\title{
Data Transmission Scheme of VANET Based on CH Selection and Switch between Clusters
}

\author{
Wei Rong ${ }^{1}$, Shen Xibing ${ }^{2}$ and Yang $\mathrm{Yi}^{3}$ \\ ${ }^{1}$ College of humanities, Qinzhou University, Qinzhou, 535000, China \\ ${ }^{2}$ College of resources and environment, Qinzhou University, \\ Qinzhou, 535000, China \\ ${ }^{3}$ College of software, Guangxi University of Science and Technology, \\ Liuzhou, 545006, China \\ E-mail:404580539@qq.com
}

\begin{abstract}
A data transmission scheme based on cluster is proposed for data transmission of VANETs. Firstly, the selection scheme of cluster head $\mathrm{CH}$ is designed. The optimal CH is selected by calculating the utility function of $\mathrm{CCHs}$, which combines node degree (NOD), available resource and mobile information of $\mathrm{CHs}$ and $\mathrm{CMs}$, including the characteristic requirements of different user applications. According to the simulation results, the proposed scheme's performance can be improved in terms of throughput, data transmission delay, successful transmission rate and overhead.
\end{abstract}

Keywords: VANET; Cluster; Data transmission; Switch; Resource

\section{Introduction}

As a special form of MANET (Mobile ad-hoc network), VANET (Vehicular ad-hoc network) provides a platform for V2VC (Vehicle-to-Vehicle Communication) and V2RC (Vehicle-to-Roadside Unit Communication). Through V2VC, information is shared between vehicles to improve traffic safety. However, due to the high speed movement of the vehicles, dynamic change of the topology structure, characteristics of the channel fading and competition mechanism of the channel in VANET, the data transmission of VANET is challenged. In the ANET, MNs (Mobile nodes) are in free distribution and form swarms, namely clusters, based on their location. Therefore, V2VC of VANET can be realized through the cluster based network structure. According to the literature [1-2], the cluster based scheme can improve the utilization of resources and enhance the network capacity. Although cluster based schemes are widely used in sensor networks $[3,4]$, the schemes designed based on the sensor network cannot be directly used in VANET due to the different characteristics of the network. To this end, many researchers have conducted theoretical studies on clusters in the VANET. The literature proposes a distributed and dynamic cluster related master $\mathrm{CH}$ (Cluster head) selection algorithm, by which the network is divided into several clusters. The algorithm uses the fuzzy logic inference strategy to predict the velocity and location information of CMs (Cluster members). In the literature [6], a novel cluster algorithm based on beacon is adopted, and the new ALM (Aggregate local mobility) scheme is introduced to construct the cluster structure. In the literature [7], the author proposes a simple cluster selection strategy that RSS (Received signal strength) is used as the cluster information index. In addition, for VANET, the literature [8] puts forward LocVSDP (Location-based service discovery protocol). LocVSDP uses cluster based facilities and provides time-sensitive and scalable network architecture with location-based service.

The above research work does not fully consider strategy mechanism of selecting CHs. In addition, the switch between clusters brings dynamic changes of the vehicle 
performance parameters, thus leading to the fact that the selected $\mathrm{CH}$ may not be the best, which will reduce the vehicle data transmission performance. For this reason, this paper proposes a novel $\mathrm{CH}$ selection algorithm for a given cluster based on three types of information including node degree, available resource of $\mathrm{CCHs}$ (Candidate $\mathrm{CHs}$ ) and velocity difference between $\mathrm{CCHs}$ and $\mathrm{CMs}$. In view of the characteristics of the user applications, the UTF (Utility function) related to CM is proposed and the utility variations accessing to different clusters are compared, so that the vehicles can be switched between different clusters.

\section{System Model}

In this paper, the VANET is considered to be applied based on many vehicles and one AP (Access point). And different clusters are assumed to be constituted by the adjacent vehicles that are close to each other in each region. For each cluster, one $\mathrm{CH}$ is selected and other vehicles are considered as CMs. CMs of a cluster have direct communication with other vehicles within the cluster, and CMs must be able to communicate with the $\mathrm{CH}$, through which the data is forwarded to AP or the vehicles in other clusters.

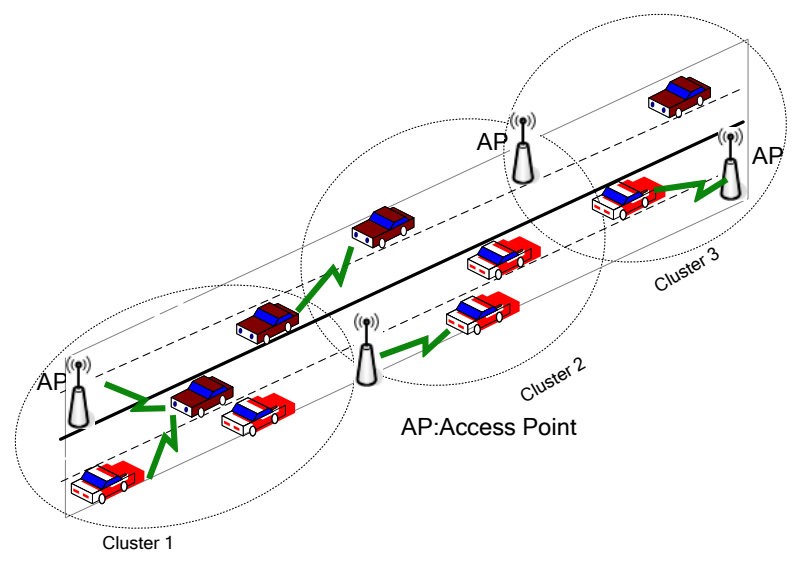

Figure 1. Network Model

As shown in Figure 1, the vehicles constitute three clusters. For the clusters that have been constituted, the optimal $\mathrm{CH}$ should be selected. In addition, the vehicles in one cluster can choose to stay within the cluster or request to come into another cluster (request for switch between clusters). And there should be a standard to determine whether to accept the request. In the third section, the selection scheme of $\mathrm{CH}$ is analyzed, and the request for switch between clusters is analyzed in the fourth section.

The communication channel model between $\mathrm{CH}$ and AP is Nakagamim-m fading channel, with channel gain $h_{1}$. Its PDF (Probability distribution function) [6] is as indicated by the formula (1).

$$
f\left(h_{1}\right)=\frac{2 m^{m}}{\Omega(d)^{m} \Gamma(m)} h_{1}^{2 m-1} \exp \left(-\frac{m}{\Omega(d)} h_{1}^{2}\right)
$$

Where, $m$ represents the Nakagamim fading parameter $(m \geq 1 / 2) . \Gamma(\cdot)$ represents the gamma function [9]. $\Omega(d)$ represents power loss of the transmission distance $d$, as indicated by the formula (2).

$$
\Omega(d)=\frac{P_{t} G_{t} G_{r} h_{t}^{2} h_{r}^{2}}{d^{\theta} L}
$$


Where, $P_{t}$ represents the transmission power, $G_{t}$ and $G_{r}$ represent the gains of transmit and receive antennas respectively, $h_{t}$ and $h_{r}$ represent the length of transmit and receive antennas respectively. $\theta$ represents the path loss index, and $L$ represents the system loss.

The communication channel model between CMs is Cascaded Nakagmai-m fading channel, 2 Cascaded. PDF[10] of channel gain $h_{2}$ is as indicated by the formula (3).

$$
f\left(h_{2}\right)=\frac{2}{h_{2} \Gamma\left(m_{1}\right) \Gamma\left(m_{2}\right)} G_{0.2}^{2.0}\left[\frac{m_{1} m_{2} h_{2}^{2}}{\Omega_{1} \Omega_{2}} \mid-\bar{m}_{1}, m_{2}\right]
$$

Where, $G_{0,2}^{2,0}(. \mid:)$ is the function of Meijer G- [11]. $\Omega_{l}=E\left[h_{l}^{2}\right]$, $m_{1}=\Omega_{l}^{2} / E\left(h_{l}-\Omega_{l}\right)^{2} \geq 0.5$, and $l=1,2$.

\section{Scheme to Select the Optimal CH}

In fact, in a cluster, $\mathrm{CH}$ plays a role in forwarding nodes for $\mathrm{CMs}$. To this end, the scheme to select the optimal $\mathrm{CH}$ should take the transmission performance of CMs into account. The next step is to analyze the scheme for selection of $\mathrm{CH}$.

\subsection{Factors Influencing Selection of $\mathbf{C H}$}

For a given cluster, to select the optimal $\mathrm{CH}$ should consider the following factors:

3.1.1. NOD (Node Degree): The $\mathrm{CH}$ in the cluster should have the ability to directly communicate with all the CMs in this cluster. In other words, $\mathrm{CH}$ should have the highest NOD in the cluster. The NOD of the vehicle ${ }^{i}$ within the cluster $m$ is marked as $N_{i}^{m}$. Obviously, $N_{i}^{m}$ is related to the number of members of the cluster. To this end, the maximum number of the members of each cluster is defined as $N^{\max }$.

3.1.2. NVD (Node velocity difference): Because both $\mathrm{CHs}$ and $\mathrm{CMs}$ are moving at a high velocity, the vehicle movement information must be considered in order to keep the topological structure of the cluster stable. Obviously, the smaller velocity difference between $\mathrm{CHs}$ and CMs makes the cluster structure more stable. Therefore, in selecting $\mathrm{CH}$, there is need to consider its velocity information.

$v_{i}^{m}$ represents the velocity of the vehicle $i$ in the cluster $m$. The average value $\Delta v$ of the velocity difference between the vehicle $i$ and CMs in the cluster $m$ is as indicated by the formula (4).

$$
\Delta v=\frac{\sum_{j \in \Omega} m,{ }_{j \neq i}\left|v_{i}^{m}-v_{j}^{m}\right|}{2 N^{m} v^{\max }}
$$

Where, $N^{m}$ and $v^{\max }$ represent the number of CMs in the cluster $m$ and the maximum velocity of $\mathrm{CMs}$

\subsubsection{AVR (Available Resource)}

In order to forward packets to $\mathrm{CM}, \mathrm{CH}$ needs to consume a certain amount of bandwidth resources. To this end, in selecting $\mathrm{CH}$, the normalized access bandwidth variable is defined, as indicated by the formula (5).

$$
B_{i}^{m, N o r}=\frac{B^{m, a v}}{B^{\max }}
$$


Where, $B^{m, a v}$ and $B^{\max }$ represent the value of the access bandwidth of the vehicle $i$ in the cluster $m$

\subsection{Performance Evaluation of CCHs}

In order to comprehensively evaluate the performance of $\mathrm{CCHs}$, the sigmoid function in Machine Learning [11] is applied and the UF (function Utility) is defined.

$\mathrm{UF}$ of $\mathrm{CCH} i$ in the cluster $m$ is as indicated by the formula (6).

$$
U_{i}^{m}=\frac{B^{m, N o r}}{1+e^{-s\left(w_{n} \frac{N_{i}}{N_{\max }}-\Delta v+w\right)}}
$$

Where, $S, w_{n}$ and $w$ are parameters of the Sigmoid function. $S$ represents the gradient of the utility curve, $w_{n}$ and $w$ jointly determine the knee in the curve.

According to the formula (6), the utility value of the cluster $m$ is calculated, and the $\mathrm{CCH}$ that has the largest utility is selected as the optimal $\mathrm{CH}$, as indicated by the formula (7).

$$
C H_{m}^{*}=\arg \max _{i} U_{i}^{m}
$$

\section{Scheme for Switch between Clusters}

For clusters with a selected $\mathrm{CH}$, the data transmission performance of $\mathrm{CMs}$ depends on the characteristics of $\mathrm{CH}$ and $\mathrm{CMs}$, and the requirements of the user applications.

Let's assume that $\mathrm{CM}_{k}$ belongs to the cluster $m$. If $\mathrm{CM}_{k}$ goes into the cluster $n$, $\mathrm{CM}_{k}$ will have the better transmission performance. To this end, $\mathrm{CM}_{k}$ may apply to enter the cluster. In this case, in the decision to accept or reject such request, the cluster $n$ needs to assess the impact of accepting the vehicle $\mathrm{CM}_{k}$ on the performance. In this section, analysis is conducted on this issue. Specifically, the task is to define access to a cluster and accept the UF of a CM.

\subsection{UF of CMs}

According to the sensitivity of time and throughput, the applications of VANET can be divided into two categories, namely, SA (Safety Application) and UIAA (User Internet Accessing Application).

\subsubsection{DSS (Delay-sensitive service)}

When the UF is established, the transmission delay should be taken into account. If the link transmission performance is poorer, there is a greater probability that transmission fails and retransmission is needed, finally leading to a longer transmission delay. Therefore, the quality of the link can be used to evaluate the transmission performance of DSS. In addition, in VANET, the communication between CMs and between CM and AP is in accordance with the protocol of IEEE 802.11 based on MAC (Media access). Some collision during the transmission may occur when many nodes transmit messages at the same time, resulting in transmission failure. To avoid such failure, the backoff and retransmission mechanism is introduced. The UF of $\mathrm{CM}_{k}$ in the cluster $m$ is $V_{k}^{m, d e}$, as indicated by the formula (8).

$$
V_{k}^{m, d e}=\beta^{d e} \frac{P_{k}^{m}}{T_{k}^{m}}
$$


Where, $\beta^{d e}$ represents the transmission efficiency of DSS; $P_{k}^{m}$ and $T_{k}^{m}$ represent the probability of successful transmission and the transmission time of $\mathrm{CM}_{k}$ in the cluster $m$ respectively. Due to the possibility that $\mathrm{CM}_{k}$ may directly communicate with $\mathrm{CH}$ and other $\mathrm{CMs}$, or communicate with $\mathrm{AP}$ through $\mathrm{CH}$, the average successful transmission probability is as indicated by the formula (9).

$$
P_{k}^{m}=p_{k}^{m, d e, d i r} P_{k}^{m, d i r}+\left(1-p_{k}^{m, d e, d i r}\right) P_{k}^{m, A P}
$$

Where, $p_{k}^{m, d e, d i r}$ represents the probability of direct transmission between $\mathrm{CM}_{k}$ and other vehicles in the same cluster. In general, $p_{k}^{m, d e, d i r}$ is far greater than 0.5. $P_{k}^{m, d i r}$ represents the probability of successful direct transmission between $\mathrm{CM}_{k}$ and CMs in the cluster $m . P_{k}^{m, A P}$ presents the probability of successful transmission between AP and $\mathrm{CM}_{k}$ through $\mathrm{CH}$. According to the channel model defined in the second section, $P_{k}^{m, d i r}$ and $P_{k}^{m, A P}$ are as indicated, respectively, by formulas (10) and (11).

$$
\begin{aligned}
& P_{k}^{m, d i r}=\operatorname{Pr}\left(\frac{E_{s}}{N_{0}} h_{1}^{2} \geq \psi_{t h 1}\right) \\
& =1-\frac{G_{1,3}^{2,1}\left[\frac{m_{1} m_{2} N_{0} \psi_{t h 2}}{\Omega_{1} \Omega_{2} E_{s}} \mid \begin{array}{c}
1 \\
m_{1}, m_{2}, 0
\end{array}\right]}{\Gamma\left(m_{1}\right) \Gamma\left(m_{2}\right)} \\
& P_{k}^{m, A P}=\operatorname{Pr}\left(\frac{E_{s}}{N_{0}} h_{1}^{2}>\psi_{t h 1}\right) \times \operatorname{Pr}\left(\frac{E_{s}}{N_{0}} h_{2}^{2}>\psi_{t h 2}\right) \\
& \left.=\left(1-\frac{\gamma\left(m, \frac{m N_{0}}{\Omega E_{s}} \psi_{t h 1}\right)}{\Gamma(m)}\right) \times\left(1-\frac{G_{1,3}^{2,1}\left[\frac{m_{1} m_{2} N_{0} \psi_{t h 2}}{\Omega_{1} \Omega_{2} E_{s}} \mid m_{1}, m_{2}, 0\right.}{}\right]\right)
\end{aligned}
$$

Where, $\gamma$ represents the incomplete gamma function.

In addition, $T_{k}^{m}$ in the formula (8) is as shown in the formula (12).

$$
T_{k}^{m}=p_{k}^{m, d e, d i r} T_{k}^{m, d i r}+\left(1-p_{k}^{m, d e, d i r}\right) T_{k}^{m, A P}
$$

Where, $T_{k}^{m, d i r}$ represents the time that $\mathrm{CM}_{k}$ requires to transmit data to other vehicles in the cluster $m$. The expression of $T_{k}^{m, d i r}$ is as indicated by the formula (13).

$$
T_{k}^{m, d i r}=D I F S+T_{R T S}+T_{C T S}+S I F S+T_{k}^{m, B O}+T_{k}^{m, \text { Data }}
$$

Where, DIFS and SIFS represent the distributed inter frame space and the short inter frame space defined in IEEE 802.11 respectively. $T_{R T S}$ and $T_{C T S}$ represent the time to transmit RTS and CTS respectively. $T_{k}^{m, B O}$ and $T_{k}^{m, \text { Data }}$ represent the backoff time and the time that $\mathrm{CM}_{k}$ in the cluster $m$ requires for data transmission.

$T_{k}^{m, A P}$ in the formula (12) represents the time that $\mathrm{CM}_{k}$ requires to transmit data to AP through $\mathrm{CH}$, as indicated by the formula (14).

$$
\begin{aligned}
T_{k}^{m, A P} & =T_{k}^{m, d i r}+\text { DIFS }+T_{R T S}+T_{C T S}+\text { SIFS } \\
& +T_{H}^{m, B O}+T_{H}^{m, \text { Data }}
\end{aligned}
$$


Let's assume that $\mathrm{CM}_{k}$ uses DSS and switches from the cluster $m$ to the cluster $n$, the add value of the UF is as indicated by the formula (15).

$$
\Delta V_{k}^{m, n, d e}=V_{k}^{n, d e}-V_{k}^{m, d e}-S_{k}^{m, n, d e}
$$

Where, $S_{k}^{m, n, d e}$ represents the SC (Signaling cost) of $\mathrm{CM}_{k}$ to switch from the cluster $m$ to the cluster $n$.

4.1.2. TSS(Throughput-Sensitive Service): In VANET, the user needs to download files, watch video data and other entertainment information. To this end, high quality of the link is needed between CMs to provide a higher throughput of transmission. The access bandwidth resources of $\mathrm{CH}$ play an important role in the communication between CMs and AP. Let's assume that the $\mathrm{CM}_{k}$ user in the cluster $m$ uses TSS, the $\mathrm{UF}$ of $\mathrm{CM}_{k}$ is as indicated by the formula (16).

$$
V_{k}^{m, t h}=\beta^{t h} F_{H, k}^{m} R_{k}^{m}
$$

Where, $\beta^{\text {th }}$ presents the transmission efficiency of TSS, $F_{H, k}^{m}$ presents the bandwidth access index of $\mathrm{CH}$, as indicated by the formula (17).

$$
F_{H, k}^{m}=\left\{\begin{array}{l}
1, B_{H}^{m, a v} \geq B_{k}^{m, r e q} \\
0, B_{H}^{m, a v}<B_{k}^{m, r e q}
\end{array}\right.
$$

Where, $B_{H}^{m, a v}$ presents the access bandwidth of $\mathrm{CH}$ in the cluster $m$, and $B_{k}^{m \text {,req }}$ presents the bandwidth required by $\mathrm{CM}_{k}$ in the cluster $m . R_{k}^{m}$ presents the average capacity of $\mathrm{CM}_{k}$ in the cluster $m$, as indicated by the formula (18).

$$
R_{k}^{m}=p_{k}^{m, t h, d i r} R_{k}^{m, d i r}+\left(1-p_{k}^{m, t h, d i r}\right) R_{k}^{m, A P}
$$

Where, $p_{k}^{m, t h, d i r}$ presents the probability that $\mathrm{CM}_{k}$ directly transmits TSS to CMs in the same cluster, usually, $p_{k}^{m, t h, d i r}$ is far less than 0.5. $R_{k}^{m, d i r}$ represents the link capacity directly related to $\mathrm{CM}_{k}$ in the cluster $m$ and $R_{k}^{m, A P}$ represents the capacity of the link from $\mathrm{CM}_{k}$ to AP.

Let's assume $C_{k j}^{m}$ represents the capacity of the link from $\mathrm{CM}_{k}$ to $\mathrm{CM}_{j}$ in the cluster $m$, its expression is as indicated by the formula (19) [12].

$$
C_{k j}^{m}=B_{k j}^{m} \log \left(1+\gamma_{k j}^{m}\right)
$$

Where, $B_{k j}^{m}$ and $\gamma_{k j}^{m}$ represent the bandwidth from $\mathrm{CM}_{k}$ to $\mathrm{CM}_{j}$ in the same cluster and SNR (Signal Noise Ratio) respectively. Therefore, $R_{k}^{m, d i r}$ is as indicated by the formula (20).

$$
R_{k}^{m, d i r}=\sum_{j \in \Omega_{m}, j \neq k} \frac{c_{k j}^{m}}{(N-1)^{m}}
$$

In addition, let's assume $C_{k H}^{m}$ represents the capacity of the link from $\mathrm{CM}_{k}$ to $\mathrm{CH}$ of the cluster $m$, and $C_{H}^{m, A P}$ represents the capacity of the link from $\mathrm{CH}$ of the cluster $m$ to AP, as indicated by formulas (21) and (22).

$$
C_{k H}^{m}=B_{k H}^{m} \log \left(1+\gamma_{k H}^{m}\right)
$$




$$
C_{H}^{m, A P}=B_{H}^{m, A P} \log \left(1+\gamma_{H}^{m, A P}\right)
$$

Where, $B_{k H}^{m}$ and $\gamma_{k H}^{m}$ represent the bandwidth from $\mathrm{CM}_{k}$ to $\mathrm{CH}$ of the cluster $m$ and SNR respectively. $B_{H}^{m, A P}$ and $\gamma_{H}^{m, A P}$ represent the bandwidth from $\mathrm{CH}$ to AP and SNR respectively. $R_{k}^{m, A P}$ is as indicated by the formula (23).

$$
R_{k}^{m, A P}=\min \left(C_{k H}^{m}, C_{H}^{m, A P}\right)
$$

The UF that switches $\mathrm{CM}_{k}$ from the cluster $m$ to the cluster $n$ in the TSS is as indicated by the formula (24).

$$
\Delta V_{k}^{m, n, t h}=V_{k}^{n, t h}-V_{k}^{m, t h}-S_{k}^{m, n, t h}
$$

Where, $S_{k}^{m, n, t h}$ represents the SC that switches $\mathrm{CM}_{k}$ from the cluster $m$ to the cluster $n$.

4.1.3. PVS (Probability of Various services): Vehicles in VANET may need to access different services at different times, in a given time zone $T$, the probabilities that $\mathrm{CM}_{k}$ of the cluster $m$ transmits delay-sensitive data and throughput-sensitive data are as indicated by formulas (25) and (26) respectively.

$$
\begin{aligned}
P_{k}^{m, d e} & =\frac{T_{k}^{m, d e}}{T} \\
P_{k}^{m, t h} & =\frac{T_{k}^{m, t h}}{T}
\end{aligned}
$$

4.1.4. Average utility of CMs: The average utility increment $\Delta V_{k}^{m, n}$ of $\mathrm{CM}_{k}$ in switching from the cluster $m$ to the cluster $n$ is as indicated by the formula (27).

$$
\Delta V_{k}^{m, n}=P_{k}^{m, d e} \Delta_{k}^{m, n, d e}+P_{k}^{m, t h} \Delta_{k}^{m, n, t h}
$$

\subsection{UF of the Cluster}

When $\mathrm{CM}_{k}$ joins the cluster $n$, the transmission performance of the cluster may change. And the increase of the cluster members will lead to the rise of cluster management and maintenance costs. Due to the movement of the vehicle, the topology of the cluster changes, so the relationship between velocity and location of $\mathrm{CM}_{k}$ should be considered.

Let's assume the velocity of $\mathrm{CM}_{k}$ is $v^{k}$, the normalized velocity difference between $\mathrm{CMs}$ and $\mathrm{CM}_{k}$ of the cluster $n$ is as indicated by the formula (28).

$$
\Delta v^{n, k}=\frac{\sum_{j \in \Omega^{n}}\left|v^{k}-v_{j}^{n}\right|}{2 v^{\max }}
$$

And the normalized average distance $\Delta d^{n, k}$ between $\mathrm{CM}_{k}$ and CMs of the cluster $n$ is as indicated by the formula (29).

$$
\Delta d^{n, k}=\frac{\sum_{j \in \Omega_{n}}\left(\left|x^{k}-x_{j}^{n}\right|^{2}+\left|y^{k}-y_{j}^{n}\right|^{2}\right)^{1 / 2}}{2 R^{\max }}
$$


Where, $\left(x_{k}, y_{k}\right)$ is the position coordinate of $\mathrm{CM}_{k}$ and $\left(x_{j}^{n}, y_{j}^{n}\right)$ is the position coordinate of $\mathrm{CM}_{j}$ in the cluster $n \cdot R^{\max }$ is the maximum transmission distance of the vehicles.

When receiving $\mathrm{CM}_{k}$, the return $\mathrm{Re}^{n, k}$ that cluster $n$ gains therefrom is as indicated by the formula (30).

$$
\operatorname{Re}^{n, k}=\beta_{d} \frac{1}{\Delta d^{n, k}}+\beta_{v} \frac{1}{\Delta v^{n, k}}
$$

Where, $\beta_{v}$ and $\beta_{d}$ represents the weights of the velocity difference and the distance difference respectively.

Let's assume the number of CMs in the cluster $n$ is $N_{n}$. According to the literature [13], the SC in charge of management and maintenance of the cluster $n$ is $\alpha N_{n}^{2}$, where $\alpha$ is a constant. If the cluster $n$ receives $\mathrm{CM}_{k}$, the $\mathrm{SC}$ is $\alpha\left(N_{n}+1\right)^{2}$, therefore, the increment $\Delta C^{n, K}$ of SC is as indicated by the formula (31).

$$
\Delta C^{n, k}=\alpha\left(\left(N_{n}+1\right)^{2}-N_{n}^{2}\right)=2 \alpha N_{n}+\alpha
$$

In taking in $\mathrm{CM}_{k}$, it is required to consider the benefits of receiving $\mathrm{CM}_{k}$ and cost increment. The utility of the cluster $n$ is as indicated by the formula (32).

$$
\Delta U^{n, k}=\operatorname{Re}^{n, k}-\Delta C^{n, k}
$$

\subsection{Selection of the Cluster}

When the vehicle $\mathrm{CM}_{k}$ is switched from the cluster $m$ to the cluster $n$, the cluster $n$ uses the formula (32) to evaluate the utility of accepting $\mathrm{CM}_{k}$. If $\Delta U^{n, k} \geq 0$, the cluster $n$ will accept the request, otherwise, it will not. More specifically, as long as $\Delta U^{n^{*}, k} \geq 0$ is met, $\mathrm{CM}_{k}$ will be switched from the cluster $m$ to the cluster $n^{*}$, where, $n^{*}=\arg \max \Delta V_{k}^{m, n}$.

\section{Simulation and Numerical Analysis}

In this section, the proposed scheme for $\mathrm{CH}$ selection and switch between clusters is simulated and compared with the scheme proposed in [5]. The simulation scenario is shown in Figure 2, where the road is $2 \mathrm{Km}$ long and there is an AP on the road with a communication radius of $800 \mathrm{~m}$, the vehicle's communication radius is $200 \mathrm{~m}$. Vehicles are randomly distributed on the road at velocities ranking from $40 \mathrm{Km} / \mathrm{h}$ to $120 \mathrm{Km} / \mathrm{h}$.

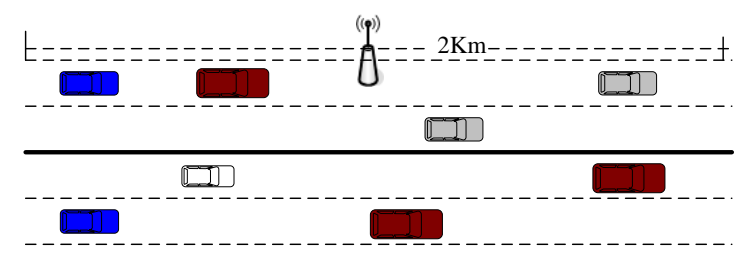

Figure 2. Simulation Scenario 


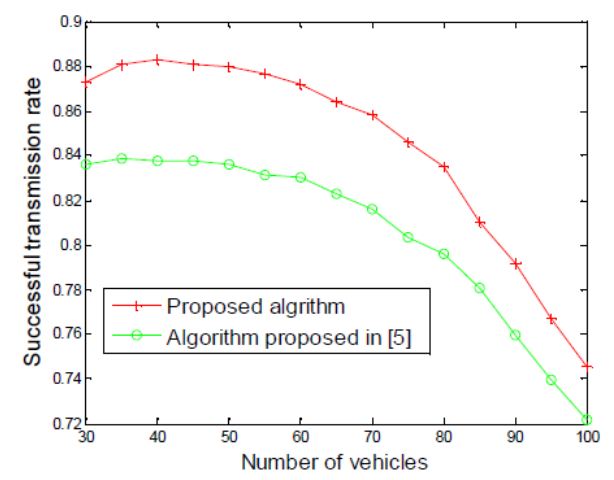

Figure 3. Successful Transmission Rate

Figure 3 shows the impact of the number of vehicles on the successful transmission rate. From the figure we can see that, with the increase of the number of vehicles, the successful transmission rate of the two algorithms drops. This is mainly due to the increase in the number of vehicles, which enhances the probability of data collision and then reduces the successful transmission rate. In addition, from the figure we can also see that, compared with the scheme proposed in [5], the algorithm proposed in this paper improves the successful transmission rate in performance. The reason is that the proposed algorithm combines a variety of factors, including channel characteristics of the physical layer, collision and bandwidth access.

Figure 4 shows the throughput charges of all vehicles along with the change in the number of vehicles. From the figure we can see that the proposed algorithm is better than that proposed by the literature [5] in performance. The reason is that the proposed algorithm considers the bandwidth between $\mathrm{CMs}$ and $\mathrm{CH}$ as well as the link information between. With the increase of the number of vehicles, the total throughput rises.

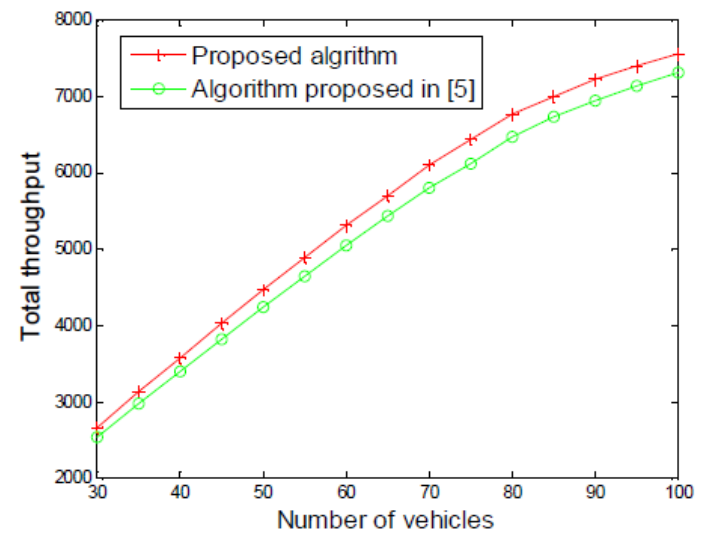

Figure 4. Throughput vs Number of Vehicles 


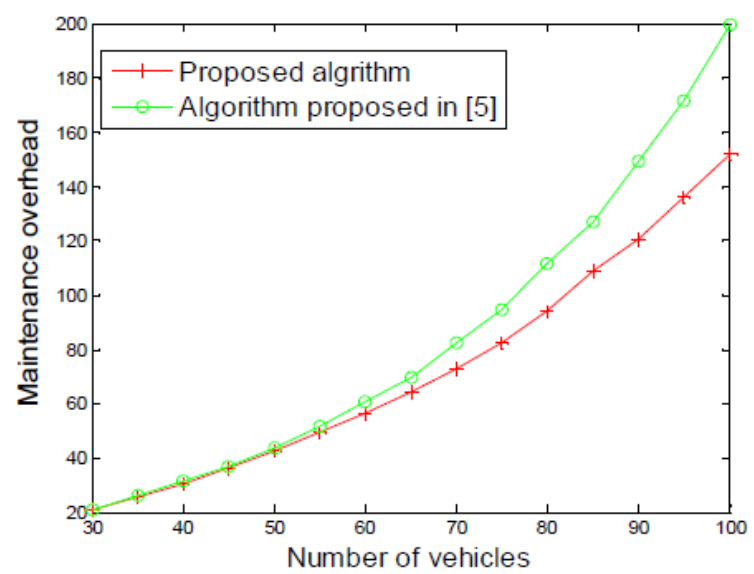

Figure 5. Maintenance Overhead vs Number of Vehicles

Figure 5 shows the variation curves of the two algorithms along with the change in the number of vehicles. From the figure we can see that the overhead of the proposed algorithm is lower than that of the algorithm proposed [5]. It is mainly because the proposed algorithm combines the velocity of vehicles, NOD and bandwidth access information in selecting $\mathrm{CH}$. With this information, the topology of the cluster is more stable, which can reduce the maintenance overhead of the cluster.

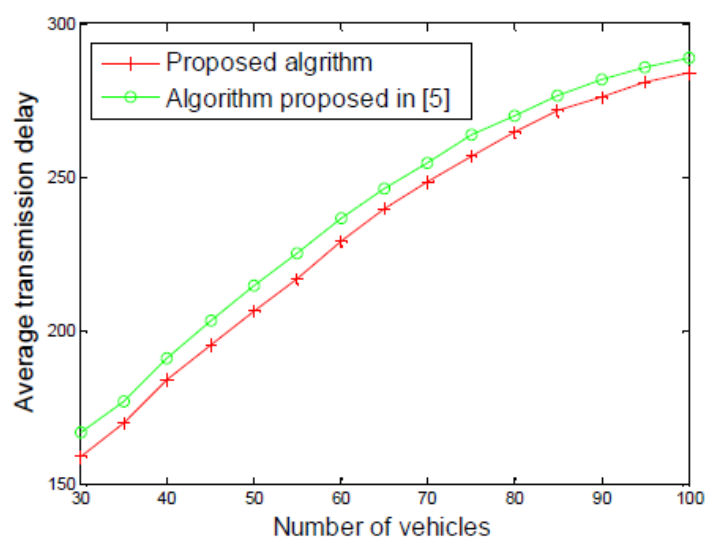

Figure 6. Average Transmission Delay vs Number of Vehicles

The average transmission delay of the two algorithms changes with the change in the number of vehicles, as shown in Figure 6. Compared with the algorithm proposed in [5], the proposed algorithm has a lower transmission delay due to the reason that the proposed algorithm combines the requirements of the applications in switching between clusters as well as the performance between clusters, and $\mathrm{CH}$ is selected through the UF to help CM switch between clusters, thus reducing the transmission delay.

\section{Conclusion}

Due to the characteristics of VANET (Vehicular ad-hoc network), including high speed movement of vehicles, channel fading, competition between channels and QoS (Quality of Service), the data transmission is challenged. To this end, the data transmission strategy VANET based on cluster is adopted with introduction of the selection scheme of $\mathrm{CH}$ (Cluster head) and the algorithm of switch between clusters. The selection scheme of $\mathrm{CH}$ combines three aspects of information including NOD 
(Node degree), access resources of $\mathrm{CCHs}$ (Candidate Cluster head) and velocity difference between $\mathrm{CCHs}$ and other $\mathrm{CMs}$ (Cluster members). In addition, QoS requirements of DSS (delay-sensitive service) and TSS (throughput sensitive service) are taken into account in switching between clusters, and the UF (Utility functions) is established to realize switch between clusters. Finally, the simulation results show that the proposed scheme significantly improves the performance in terms of data transmission delay, overhead, successful transmission rate and throughput.

\section{References}

[1] D. Jiang, X. Ying and Y. Han, "Collaborative multi-hop routing in cognitive wireless networks", Wireless Personal Communications, (2015), pp. 1-23.

[2] Z. Lv, A. Tek and F. Da Silva, "Game on, science-how video game technology may help biologists tackle visualization challenges", PloS one, vol. 8, no. 3, (2013), p. 57990.

[3] D. Jiang, Z. Xu and Z. Lv, "A multicast delivery approach with minimum energy consumption for wireless multi-hop networks", Telecommunication Systems, (2015), pp. 1-12.

[4] C. Fu, P. Zhang and J. A. Jiang, "Bayesian approach for sleep and wake classification based on dynamic time warping method", Multimedia Tools and Applications, (2015), pp. 1-20.

[5] Z. Lv, "Wearable smartphone: Wearable hybrid framework for hand and foot gesture interaction on smartphone, Computer Vision Workshops (ICCVW), 2013 IEEE International Conference on IEEE, (2013), pp. 436-443.

[6] Y. Lin, J. Yang and Z. Lv, "A Self-Assessment Stereo Capture Model Applicable to the Internet of Things", Sensors, vol. 15, no. 8, (2015), pp. 20925-20944.

[7] J. Yang, S. He and Y. Lin, "Multimedia cloud transmission and storage system based on internet of things", Multimedia Tools and Applications, (2015), pp. 1-16.

[8] Z. Lv, T. Yin and Y. Han, "WebVR——web virtual reality engine based on P2P network", Journal of Networks, vol. 6, no. 7, (2011), pp. 990-998.

[9] J. Yang, S. He and Y. Lin, "Multimedia cloud transmission and storage system based on internet of things", Multimedia Tools and Applications, (2015).

[10] C. Guo, X. Liu and M. Jin, "The research on optimization of auto supply chain network robust model under macroeconomic fluctuations", Chaos, Solitons \& Fractals, (2015).

[11] X. Li, Z. Lv and J. Hu, "XEarth: A 3D GIS Platform for managing massive city information", Computational Intelligence and Virtual Environments for Measurement Systems and Applications (CIVEMSA), 2015 IEEE International Conference on. IEEE, (2015), pp. 1-6.

[12] J. Yang, B. Chen and J. Zhou, "A Low-Power and Portable Biomedical Device for Respiratory Monitoring with a Stable Power Source", Sensors, vol. 15, no. 8, (2015), pp. 19618-19632.

[13] G. Bao, L. Mi, Y. Geng and K. Pahlavan, "A computer vision based speed estimation technique for localiz ing the wireless capsule endoscope inside small intestine", 36th Annual International Conference of the IEEE Engineering in Medicine and Biology Society (EMBC), (2014).

[14] X. Song and Y. Geng, "Distributed community detection optimization algorithm for complex networks", Journal of Networks, vol. 9, no. 10, (2014), pp. 2758-2765.

[15] D. Jiang, X. Ying and Y. Han, "Collaborative multi-hop routing in cognitive wireless networks", Wireless Personal Communications, (2015), pp. 1-23.

[16] J. Hu and Z. Gao, "Modules identification in gene positive networks of hepatocellular carcinoma using Pearson agglomerative method and Pearson cohesion coupling modularity", Journal of Applied Mathematics, 2012 (2012).

[17] D. Jiang, Z. Xu and Z. Chen, "Joint time-frequency sparse estimation of large-scale network traffic", Computer Networks, vol. 55, no. 15, (2011), pp. 3533-3547.

[18] J. Hu, Z. Gao and W. Pan, "Multiangle Social Network Recommendation Algorithms and Similarity Network Evaluation", Journal of Applied Mathematics, 2013 (2013).

[19] M. Zhou, G. Bao, Y. Geng, B. Alkandari and X. Li, "Polyp detection and radius measurement in small intestine using video capsule endoscopy”, 2014 7th International Conference on Biomedical Engineering and Informatics (BMEI), (2014). 


\section{Authors}

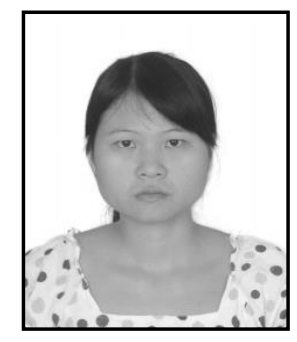

Wei Rong, she graduated from Guangxi University of science and technology, computer science and technology professional. Received master degree in computer applications. Now she is a senior engineer at Qinzhou University in Guangxi, humanities. Her main research direction is computer graphics and computer network security and system analysis. She has published academic papers. 